P. FORGACS

Z. HORVATH

L. PALLA
TOWARDS COMPLETE INTEGRABILITY OF THE SELF-DUALITY EQUATIONS

Fungarian Fcademy of Sciences

CENTRAL

RESEARCH

INSTITUTE FOR

PHYSICS

BUDAPEST 


\title{
TOWARDS COMPLETE INTEGRABILITY \\ OF THE SELF-DUALITY EQUATIONS
}

\author{
P. Forgács \\ Central Research Institute for Physics \\ H-1525 Budapest 114, P.O.B.49, Hungary \\ and
}

Z. Horváth and L. Palla

Institute for Theoretical Physics Roland Eötvös University

H-1088 Budapest, Hungary 


\section{ABSTRACT}

A solution generating method for the self-duality and the Bogomolny equations is given. We point out the existence of an infinite parameter invariance group of these equations.

\section{АННОТАЦИЯ}

Разработан метод генерирования решений уравнений Богомольного и самодуальности. Показано супествование группы инвариантности этих уравнений с бесконечным числом параметров.

\section{KIVONAT}

Az SU(2) öndualitási és a Bogomolny egyenletek megoldásainak generálására alkalmas módszert fejlesztünk ki. Megmutatjuk, hogy ezen egyenletek rendelkeznek egy végtelen paraméteres unvariancia csoporttal. 
During the past few years a great deal of progress has been made in the study of the self-duality /SDE/ and the Bogomolny equations of $S U(N)$ gauge theories. Recently, Atiyah et al. I have constructed the general n-instanton solution of the SDE using algebraic geometry. However, in Ref. 2 it was shown that there exist non instanton type solutions of the SDE as well which have finite action and, in general, non integer Pontryagin number. Furthermore, it is known that the SDE in the static case are equivalent to the Bogomolny equations which describe multimonopole solutions. It appears quite difficult to classify and construct explicitly these interesting solutions by the geometric methods of Atiyah et al. ${ }^{1}$ alone, and we think it would be helpful to develop solution generating methods for these equations. The construction of such a method is the main point of our paper. Applying these ideas we actually found multiply charged axially symmetric monopoles ${ }^{3}$.

There are some hints that the SDE are completely integrable; such as the existence of infinitely many / nonlocal / conservation laws ${ }^{4,5}$, and Bäcklund transformations ${ }^{4,6}$. In this paper we show yet other indications: the existence of an infinite parameter invariance group and an "inverse scattering" problem capable of generating a huge family of solutions. It has also been proved earlier in Ref. 7 that the SDE can be reduced in a special case to the Ernst equation ${ }^{8}$ of general relativity for which all the above mentioned properties are known.

As a first step, we show that the SDE can be interpreted as the vanishing of the invariant trace of the curvature tensor of a hermitian metric with a special cylindrical symmetry defined 
on an $\mathrm{N}+2-\mathrm{dimensional} \mathrm{complex} \mathrm{manifold:}$

$$
\begin{aligned}
& d s^{2}=d y d \bar{y}+d z d \bar{z}+g_{\alpha \bar{\beta}}(y, \bar{y}, z, \bar{z}) d \xi^{\alpha} d \overline{\xi^{\beta}}=\hat{g}_{\alpha \bar{b}} d z^{a} \overline{d z^{b}} \\
& z_{1}=y=x_{1}+i x_{2}, z_{2}=x_{3}+i x_{4}, z^{2+\alpha}=\xi^{\alpha}, \alpha=1, \ldots N
\end{aligned}
$$

For the curvature tensor of this metric the hermiticity of $\hat{g}$ implies that $^{9}$

$$
\mathrm{R}_{\text {bcd }}^{\mathrm{a}}=\mathrm{R}_{\text {bed }}^{\mathrm{a}}=0 \quad \forall \mathrm{a}, \mathrm{b}, \mathrm{c}, \mathrm{d},
$$

and the nonvanishing elements are $R_{2+\beta,}^{2+\alpha} b$ /together with their complex conjugates/ for $a, b=1,2: R_{2+\beta}^{2+\alpha} b=\partial_{\vec{a}}\left[\left(\partial_{b} g\right) g^{-1}\right]_{\beta a}$ $/$ from now on we suppress the indices $\alpha, \beta /$. Now we impose the following covariant equation on the curvature

$$
\hat{g}^{\bar{a} b} R_{\bar{a} b}=\sum_{a=1,2}\left(g, a g^{-1}\right), \bar{a}=\left(g, y g^{-1}\right)_{, \bar{y}}+\left(g, z g^{-1}\right)_{, \bar{z}}=0
$$

This is the central equation of our interest. Next, we connect this equation to the self-duality equations of an $S U(N)$ gauge the. ory by assuming that $\operatorname{detg}=I$ and $g=D^{+} D$ where $D \in S L(N, C)$. Indeed, defining the gauge vector potentials as $B_{a}=-D, a^{-1}$, $B_{\bar{a}}=D^{+-1}\left(D^{+}\right), \bar{a} / a=1,2 /$ we have $F_{a b}=F_{\bar{a} \bar{b}}=0$ and $F_{a b}=$ $=-D^{+-1}\left(g, g_{a} g^{-1}\right), b^{+}, i \cdot e$. the SDE: $F_{y \bar{y}}+F_{z \bar{z}}=0$ are really equivalent to $/ 2 /$. In this formalism a gauge transformation is defined as $D \rightarrow G D, D^{+} \rightarrow D^{+} G^{+}, G \in S U(N)$, i.e. $g$ is gauge invariant. The connection we found between the SDE and the geometry of a complex manifold makes it possible to find the invariance transformations of the SDE. It is easy to see that the "external coordinate transformations" leaving both the form of the metric in $/ I /$ and the value of detg invariant: $g \rightarrow \Omega(y, z) g \Omega^{+}$ with $S(y, z) \in S L(N, C)$ constitute a group of invariance transformations for eq./2/. This is the geometrical meaning of the 
invariance transformations of the SDE /2/ investigated in Refs. 4,10 .

In the case of $S U(2) /$ i.e. $N=2 /$ the geometrical picture can be used to obtain an alternative form of the SDE exhibiting a new group of invariance transformations. To this end we recall that for $\mathrm{N}=2$ adopting the

$$
g=\frac{1}{\phi}\left(\begin{array}{cc}
\phi^{2}+\rho \bar{s} & -\bar{s} \\
-s & 1
\end{array}\right)
$$

$/ \phi$ real, $\rho$ complex / parametrisation one obtains from $/ 2 /$ the Yang equations in the R-gauge ${ }^{11}$

$$
\begin{aligned}
& \phi \nabla \bar{\nabla} \phi-\nabla \phi \bar{\nabla} \phi+\nabla_{\xi} \bar{\nabla}_{\bar{\rho}}=0, \\
& \bar{\nabla}\left(\phi^{-2} \nabla_{\xi}\right)=0, \quad \nabla\left(\phi^{-2} \bar{\nabla} \bar{s}\right)=0, \\
& \nabla \equiv\left(\partial_{y}, \partial_{z}\right), \quad \bar{\nabla}=\left(\partial_{\bar{y}}, \partial_{\bar{z}}\right)
\end{aligned}
$$

We observe that $/ 3 \mathrm{~b}-\mathrm{c} /$ are identically satisfied if we introduce a new function $\omega$ by the definition

$$
-\phi^{-2} \tilde{\nabla}_{\rho}=\bar{\nabla} \bar{\omega} \quad, \quad \tilde{\nabla} \equiv\left(\partial_{z},-\partial_{y}\right)
$$

In terms of $\phi, \omega, \bar{\omega}$ the SDE take the following form

$$
\phi \nabla \bar{\nabla} \phi-\nabla \phi \bar{\nabla} \phi+\phi^{4} \nabla \omega \bar{\nabla} \bar{\omega}=0 ; \quad \nabla\left(\phi^{2} \bar{\nabla} \bar{\omega}\right)=0 ; \quad \bar{\nabla}\left(\phi^{2} \nabla \omega\right)=0
$$

Now with the aid of $\phi, \omega, \bar{w}$ it is possible to construct such a hermitian matrix $\tilde{\mathrm{g}}$ :

$$
\tilde{g}=\left(\begin{array}{cc}
\phi & -\phi \omega \\
-\phi \bar{\omega} & \phi \omega \bar{\omega}-\phi^{-1}
\end{array}\right)
$$

with $\operatorname{det} \tilde{g}=-1$ that eq./2/ for $\widetilde{g}$ yields eqs./4/. We remark that eqs./4/ can be interpreted as the SDE for an $S U(1,1)$ gauge theory, however, this not necessary since the introduction of $w$ can be viewed as a reparametrisation of the original SU (2) theory. 
Therefore, there exists an another "coordinate transformation" leaving det $\tilde{g}$ and the form of $\hat{g} /$ see $/ / /$ built with the aid of $\tilde{\mathrm{g}}$ invariant: $\tilde{\mathrm{g}} \rightarrow \mathrm{A}(\mathrm{y}, \mathrm{z}) \tilde{\mathrm{g}} \mathrm{A}^{+} ; \mathrm{A}(\mathrm{y}, \mathrm{z}) \in \mathrm{SL}(2, \mathrm{C})$. While $\mathrm{A}(\mathrm{y}, \mathrm{z})$ acts simply on $\widetilde{E}$. it produces a nonlinear action on $\phi, \rho, \bar{\rho}$ obtained by solving in a suitable way the system of equations connecting the $(\phi, \omega, \bar{\omega})$ and $(\phi, \rho, \bar{\rho})$ sets. The action of an $A(y, z)$ transformation defined this way on $\phi, \rho, \bar{\rho}$ is not an $\Omega(y, z)$ covariant expression and, therefore, the product of an $A$ and $\Omega$ transformation is not contained in any of these two groups. Thus the repeated applications of these two transformations generate an infinite parameter invariance group of eq. /2/. This group is very important for studying the solutions of $/ 2 /$, in fact, without mentioning the existence of this group, it was used in Ref.10 to generate the infinite hiererchy of Ansatze of Atiyah and Ward ${ }^{1}$. The existence of an infinite number of / nonlocal / conservation laws for the SDE ${ }^{4,5}$ is the consequence of the existence of this infinite parameter group ${ }^{12}$.

Recently, several authors derived Bäcklund transformations $^{4,5}$ for eq. $/ 2 /$. This fact together with the existence of the aforementioned infinite number of conservation laws leads one naturally to attempt the derivation of an inverse scattering problem for this equation. To this end, we rewrite eq. /2/ introducing the quantities $A_{a}=g, a g^{-1}, / a=1,2 /$ :

$$
\partial_{z} A_{y}-\partial_{y} A_{z}+\left[A_{y}, A_{z}\right]=0, \quad\left(A_{y}\right)_{\bar{y}}+\left(A_{z}\right)_{\bar{z}}=0
$$

which may be expressed by the closed ideal of 4-forms spanned by the forms $\alpha_{i}$ defined as 


$$
\begin{aligned}
& \alpha_{1}=\left(d A_{1} \wedge d z_{1}+d A_{2} \wedge d z_{2}+\left[A_{1}, A_{2}\right] d z_{2} \wedge d z_{1}\right) \wedge d \bar{z}_{1} \wedge d \bar{z}_{2} \\
& \alpha_{2}=\left(d A_{1} \wedge d \bar{z}_{2}-d A_{2} \wedge d \bar{z}_{1}\right) \wedge d z_{1} \wedge d z_{2}
\end{aligned}
$$

We determine an inverse scattering problem for eqs. /5/ using the notion of prolongation structures. Indeed, using the method of Ref.13 one obtaines a linear 3-form $\tau$ that prolongs the ideal spanned by $\alpha_{i}$ :

$$
\begin{gathered}
\tau=d \psi \wedge\left\{d \bar{z}_{1} \wedge d \bar{z}_{2}+\lambda\left(d z_{1} \wedge d \bar{z}_{1}+d z_{2} \wedge d \bar{z}_{2}\right)+\lambda^{2} d z_{1} \wedge d z_{2}\right\}- \\
-d z_{2} \wedge d \bar{z}_{1} \wedge\left(d \bar{z}_{2}-\lambda d z_{1}\right) A_{2} \psi+d z_{1} \wedge d \bar{z}_{2} \wedge\left(d \bar{z}_{1}+\lambda d z_{2}\right) A_{1} \psi
\end{gathered}
$$

where $\lambda$ is an arbitrary constant parameter. If we section this 3-form onto the solution manifold of eqs. /5/ we obtain the inverse scattering equations ${ }^{14}$ :

$$
\left(\lambda \partial_{\bar{z}}+\partial_{y}\right) \psi=A_{y} \psi, \quad\left(-\lambda \partial_{\bar{y}}+\partial_{z}\right) \psi=A_{z} \psi
$$

From these equations we immediately see that $\psi(\lambda=0, y, \bar{y}, z, \bar{z})=$ $=g(y, \bar{y}, z, \bar{z})$. It is straightforward to obtain the transform mation properties of $\psi$ under the coordinate transformations discussed above; if $g$ is transformed by $\Omega(y, z): g^{\prime}=\Omega g \Omega^{+}$ then $\psi^{\prime}=\Omega \psi F^{+}$where $F=\Omega(y+\bar{\lambda} \bar{z}, z-\bar{\lambda} \bar{y})$. The other remarkable property of $/ 6 /$ is that by expanding $\psi$ in powers of $\lambda$

$$
\psi=\sum_{n} \lambda^{n} g^{(n)} \quad \text { we obtain the infinitely many }
$$

conservation laws of Refs.4,5.

$$
\nabla\left(g^{(0)-1} g^{(n+1)}\right)=\left(g^{(0)-1} \overline{\widetilde{\nabla}} g^{(0)}+\overline{\widetilde{\nabla}}\right)\left(g^{(0)-1} g^{(n)}\right)
$$

Carrying out this expansion in the transformation law of $\psi$ one obtaines that the infinitely many conserved quantities form an 
infinite dimensional representation of the invariance group $\Omega$. In the case of SU(2) we obviously have two similar sets of equations corresponding to the possibility of working with either $g$ or $\tilde{\mathrm{g}}$ matrices. This means that in SU (2) the SDE have yet another set of infinite conservation laws. This underlines the fact that our inverse scattering equations are intimately connected to the existence of the infinite parameter invariance group of eq. $/ 2 /{ }^{12}$, to be contrasted with the equations of Ref.15 which are connected with a hidden $O(4)$ symmetry as it was shown by Pohlmeyer ${ }^{4}$.

At this point we would like to make contact with certain 4-dimensional nonlinear sigma models. If $g$ is the matrix describing a 4-dimensional principal sigma model then the field equations take the form

$$
\sum_{a=1,2}\left\{\left(g, a g^{-1}\right), \bar{a}+\left(g, \bar{a} g^{-1}\right), a\right\}=0
$$

Now if $g$ is a unitary / or quasiunitary / matrix - i.e. we are working with an $S U(N)$ or $S U(N, M)$ principal sigma model - then a sufficient / but not necessary / condition for $g$ to solve /7/ is the satisfaction of eq. $/ 2 /$. This equation in these models may play a role similar to that of the SDE in gauge theories. As we derived the inverse scattering equations / $/ 6 /$ directly from $/ 2 /$, /6/ can be used for this class of solutions of these sigma models as well. / Note this argument remains valid for any reduction of these models./

In what follows, we discuss how one can use the inverse scattering equations $\% / 6 /$ for generating new solutions of $/ 2 / \mathrm{re-}$ stricting our attention to the construction of "soliton" solutions. / It was shown that the inverse scattering problems can be connected 
with the solutions of / matrix / Riemann problems, and this aproach defines the soliton solutions with $G(\lambda)=116 /$. The process we follow is the generalization of the method of Mikhailov and Zakharov ${ }^{16}$ devised for 2-dimensional sigma models.

We suppose that a $\psi_{\mathrm{o}}(\lambda, \mathrm{y}, \overline{\mathrm{y}}, \mathrm{z}, \overline{\mathrm{z}})$ solution of $/ 6 /$ is known in the case of an initial $g_{0}$ solution of $/ 2 /$, and look for new solutions of $/ 6 /$ in the form $\psi=\chi(\lambda) \psi_{0} . . /$ This implies, that $g=X(0) g_{0}$ is the new solution of $/ 2 / \%$. In the case of the SDE the hermiticity of $\mathrm{g} /$ or $\tilde{\mathrm{g}}$ for SU(2) / imposes a very important restriction on the analytical properties of $\chi$ in the complex $\lambda$ plane: $X(\lambda)=g \chi^{+-1}\left(-\bar{\lambda}^{-1}\right) g_{0}^{-1}$.

Motivated by this we look for $\chi(\lambda)$ in the form ${ }^{17}$

$$
X(\lambda)=I+\sum_{k=1}^{n} \frac{R_{k}}{\lambda-\mu_{k}}
$$

where $R_{k}, \mu_{k}$ are independent of $\lambda$ and $\mu_{k}$ is any solution of $\mu_{k} \tilde{\nabla}_{\mu_{k}}+\nabla_{\mu_{k}}=0 \quad$ / for $\chi^{-1}(\lambda)$ we assume a similar form with $R_{k}$ replaced by $S_{k}$ and $\mu_{k}$ by $\tilde{\mu}_{k}=-\bar{\mu}_{k}^{-I} /$. Solving the equations for $R_{k}, S_{k}$ emerging from /6/ we finally obtain the new solution :

$$
g_{a b}=\prod_{k=1}^{n}\left|\mu_{k}\right|\left(\left(g_{0}\right)_{a b}-\sum_{k, r}\left(\mu_{k} \bar{\mu}_{r}\right)^{-1} \Gamma_{r k}^{-1} \bar{N}_{a}^{(r)} N_{b}^{(k)}\right)
$$

where $\Gamma^{\mathrm{kr}}=\left(1+\mu_{\mathrm{k}} \bar{\mu}_{\mathrm{r}}\right)^{-1} \mathrm{~m}_{\mathrm{d}}^{(\mathrm{k})}\left(\mathrm{g}_{0}\right)_{\mathrm{dc}} \overline{\mathrm{m}}_{\mathrm{c}}^{-(\mathrm{r})}$ and $\mathrm{N}_{\mathrm{a}}^{(\mathrm{k})}=\mathrm{m}_{\mathrm{b}}^{(\mathrm{k})}\left(\mathrm{g}_{0}\right)_{\mathrm{ba}}$ with $m_{b}^{(k)}=M_{c}^{(k)} \psi{ }_{0}^{-1}\left(\mu_{k}, y, \bar{y}, z, \bar{z}\right)_{c b}$ and $M_{c}^{(k)}=M_{c}^{(k)}\left(y / \mu_{k}-\bar{z}, z \mu_{k}+\bar{y}, \mu_{k}\right)$ but otherwise arbitrary vectors.

It is possible to show that $\operatorname{detg}=(-1)^{\mathrm{n}} \operatorname{detg}_{0}$, there- 
fore if we start with a $g_{0}$ having $\operatorname{detg}_{0}=1$ then taking an even number of poles yields a $g$ matrix that can be interpreted in the $\phi, \varsigma, \bar{\rho}$ formalism, while taking an odd number of poles yields a $g$ that can be interpreted in the $\phi, \omega, \bar{\omega}$ formalism. The method we just described yields an abundance of new solutions, as an illustration, we show here how the 'tHooftWitten instantons in SU(2) emerge from this process by suitably choosing the arbitrary functions $\mathrm{M}_{\mathrm{C}}^{(\mathrm{k})}$. We find it more convenient to work in the $\phi, \omega, \bar{\omega}$ formalism and choose for the starting / vacuum / solution $\phi_{0}=1, \omega_{0}=\bar{\omega}_{0}=-1$. Furthermore, to preserve the sign of the determinant we assume two poles for the one instanton $\mu_{1}=-z^{-1} \bar{y}$ and $\mu_{2}=y^{-1}(\bar{z}+5) / b$ is a constant parameter /. It is important to realise that in the final expression for $\chi$ or $g$ it is possible to carry out the $b \rightarrow 0$ limit. Indeed, choosing $m_{a}^{(1)}=\left(0, m\left(z^{-1} R^{2},-z^{-1} \bar{y}\right)\right)$ and $m_{a}^{(2)}=\left(\bar{b} y^{-1}\left(R^{2}+\bar{b} z\right),-\Lambda^{2}(2 y)^{-1}(\bar{z}+\bar{b})\right)$ respectively, with $\mathrm{R}^{2}=\mathrm{y} \overline{\mathrm{y}}+\mathrm{z} \overline{\mathrm{z}}$ and arbitrary $\Lambda^{2}$ and $m\left(\mathrm{z}^{-1} \mathrm{R}^{2},-\mathrm{z}^{-1} \bar{y}\right)$ from $/ 9 /$ we finally obtain in the $b \rightarrow 0$ limit for the one instanton $\phi=1+\Lambda^{2} / R^{2}, \omega=\bar{w}=-\phi^{-1}$ - Proceeding in a similar way one can prove that it is possible to iterate this "two-pole" step $\mathrm{N}$ times leading to

$$
\Psi^{(N)}=\left(\begin{array}{cc}
\Psi_{N} & 1 \\
1 & 0
\end{array}\right)
$$

$\Psi_{N}=1+\sum_{i=1}^{N} \frac{\Lambda_{i}^{2}}{2 R_{i}^{2}}\left(\frac{\bar{y}+\bar{a}_{i}}{\lambda\left(z+b_{i}\right)+\bar{y}+\bar{a}_{i}}+\frac{\bar{z}+\bar{b}_{i}}{\bar{z}_{i}+\bar{b}_{i}-\lambda\left(y+a_{i}\right)}\right)$

which yields at $\lambda=0$ the $5 \mathrm{~N}$ parameter multiinstanton solutions of 'tHooft and Witten.

This method looks rather promising and it is reasonable to expect that one can find all finite action solutions of the 
SDE and the most general family of multimonopole configurations carrying out the procedure outlined in this paper. 
REFERENCES

1. M.F. Atiyah, V.G. Drinfeld, N.J. Hitchin and Yu.I. Manin, Phys. Lett. 65A, $185 / 1978 /$; M.F. Atiyah and R.S. Ward, Commun. Math. Phys. 52, $117 / 1977 /$.

2. P. Forgác3, Z. Horváth and L. Palla, CRIP-preprint, Budapest, KFKI-1980-60, to be published in Phys. Rev. Lett.

3. P. Forgács, Z. Horváth and L. Palla, CRIP-preprint, Budapest, KFKI-1980-122, to be published in Phys. Lett.B.

4. K. Pohlmeyer, Commun. Math. Phys. I2, 37 /1980/.

5. M.K. Prasad, A. Sinha and L.L. Wang, Phys. Lett. 87B, $237 / 1979 /$.

6. M.K. Prasad, A. Sinha and L.L. Wang, Phys. Rev. Lett. 43, $750 / 1979 /$.

7. P. Forgács, Z. Horváth and L. Palla, Phys. Rev. Lett. 45 , $505 / 1980 /$.

8. F.J. Ernst, Phys. Rev. 167, $1175 / 1968 /$.

9. J. Morrow and K. Kodaira, Complex Manifolds / Holt, Rinehart and Winston, Inc., New York, 1971/.

10. E.F. Corrigan, D.B. Fairlie, R.G. Yates and P. Goddard, Commun. Math. Phys. 58, $223 / 1978 /$.

11. C.N. Yang, Phys. Rev. Lett. 38, $1377 / 1977 /$.

12. P. Forgács, Z. Horváth and L. Palla to be published in Nukleonika. It was shown in this paper that a similar infinite parameter invariance group exists even for SU(N) , See also:P. Forgács, Z. Horváth and L. Palla, ITP-Budapest Report No. $394 / 1980 /$. 
13. H.C. Morris, J. Math. Phys. 21, $327 / 1980 /$.

14. The same equations were obtained using different methods by G. Sartori, Nuovo Cimento 56A, 73 /1980/; L.L. Wang, BNL- 27617 preprint, Conference on Theoretical Particle Physics, January 5-14. 1980, Guangzhou / Canton /. A.A. Belavin and V.E. Zakharov, Phys. Lett. 73B, $53 / 1978 /$.

16. V.E. Zakharov and A.V. Mikhailov, Zh. Eksp. Teor. Fiz. 74, 1953 /1978/ / Sov. Phys. JETP 47, 1017 /1978/ /. 17. V.A. Belinski and V.E. Zakharov, Zh. Eksp. Teor. Fiz. 75, 1953 /1978/ / Sov. Phys. JETP 48, 985 /1978/ /; Zh. Eksp. Teor. Fiz. 77, 3 /1979/ / Sov. Phys. JETP 50, 1 /1979/ /. 


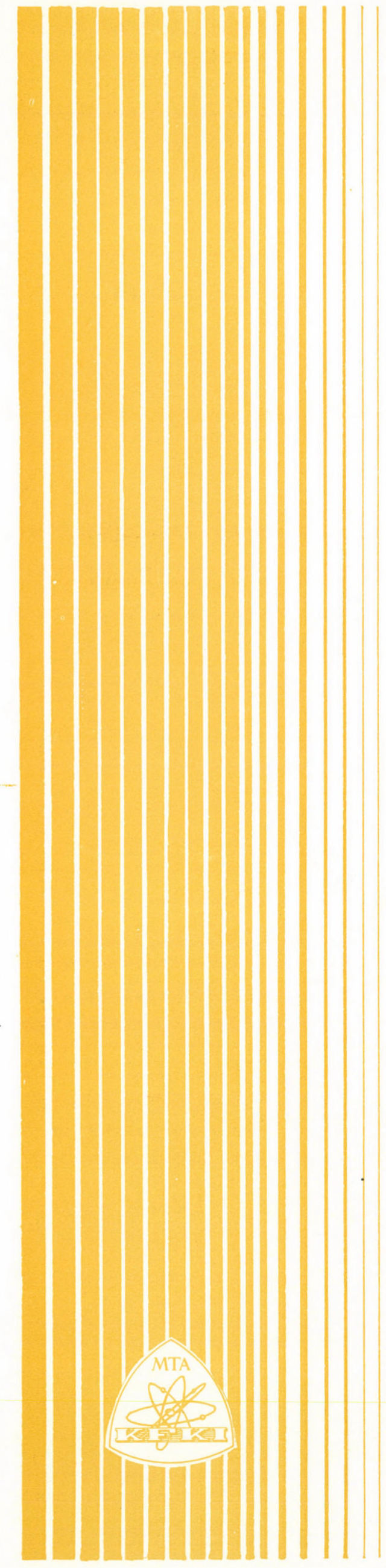

Kiadja a Központi Fizikai Kutató Intézet Felelõs kiadó: Szegõ Károly

Szakmai lektor: Hraskó Péter Nyelvi lektor: Hasenfratz Anna

Példányszám: 215 Törzsszám: 81-61 Készült a KFKI sokszorositó üzemében Felelôs vezetõ: Nagy Károly Budapest, 1981. január hó 